\title{
Does amantadine have a protective effect against COVID-19?
}

\author{
Albert Cortés Borra \\ Vall d'Hebron Hospital, Barcelona, Spain
}

The SARS-CoV-2 pandemic is causing the scientific community to look for the best treatment to fight the virus. Studies are being carried out worldwide with this purpose looking into antiviral drugs, antibiotics, antimalarials, and anticoagulants. All these investigations are in their early stages due to the nature of the pandemic, which has spread very rapidly since the first case was reported on 17 November, 2019 [1]. This means that there are no studies available that could give evidence of the most appropriate treatment for SARS-CoV-2.

Although authors such as Rodón et al. have indicated a cytotoxic effect on Vero E6 cells exposed to a fixed concentration of SARS-CoV-2 in the presence of decreasing concentrations of amantadine [2], Rejdak et al. described a series of 22 neurological patients treated with amantadine: all the patients were tested after reported person-to-person contact with SARS-CoV-2 infected subjects, and had viral infection confirmed with a real-time reverse transcription polymerase chain reaction test for the qualitative detection of nucleic acid from SARS-CoV-2 in upper and lower respiratory specimens. All of them had spent two weeks in quarantine since their documented exposure, and none developed clinical manifestations of infectious disease [3], or Aranda that exposes the hypothesis that amantadine blocks the viroporine channel of COVID-19, preventing the release of the viral nucleus into the cell cytoplasm [4] and Tipton repurposing other medications, especially those with known antiviral properties such as amantadine and memantine.

These medications are inexpensive, widely used, and have well-known side effect profiles that are relatively mild compared to other potential COVID-19 treatments such as hydroxychloroquine [5].

The reason for this letter to the editors is to present the case of a 75-year-old woman, with Parkinson's Disease of 16 years' duration, treated with opicapone, $50 \mathrm{mg} /$ day (Ongentys $\bullet$ ), pramipexol, $2.1 \mathrm{mg} /$ day (Mirapexin •), levodopa, 1,000 mg/day, benserazide, $250 \mathrm{mg} /$ day (Madopar •) and amantadine, $100 \mathrm{mg} /$ day, with a history of hyperthyroidism treated with levothyroxine, $25 \mathrm{mg}$ /day (Euthyrox •), and stomach cancer five years ago treated with surgery (Billroth II gastrectomy) and pre- and post-surgical chemotherapy, now well controlled with no cancer recurrence.

The husband of this patient, after seven days with fever with an unusual sporadic cough, was diagnosed by a positive COVID-19 PCR test with bilateral pneumonia that was the reason for hospital admission and resulted in his death.

The patient, 45 days after her husband's death, had not had any symptoms related to COVID-19, without fever, cough or anosmia, and was being looked after at home by her family. Remaining isolated without going outdoors and maintaining only contact with their direct caregivers, both had negative COVID-19 PCR tests.

Having seen the previously cited articles which hypothesise that amantadine may have a protective effect against the coronavirus $[6,7]$, this patient has been taking amantadine for seven months.

Is it possible that she was not infected with the coronavirus despite having lived with her husband with COVID-19 symptoms for seven days, even sharing a bed, with the exposure to coughing and aerosols that this produces? May amantadine work by inhibiting coronavirus infection?

For this reason, it is advisable, as the authors recommend $[4,5]$, that more studies be carried out with patients with Parkinson's Disease who are on amantadine treatment and have been directly exposed to SARS-CoV-2, in order to demonstrate the hypothesis that they put forward, and which I second.

\section{References:}

1. Zhu Na, Zhang D, Wang W, et al. A Novel Coronavirus from Patients with Pneumonia in China, 2019. New England Journal of Medicine. 2020; 382(8): 727-733, doi: 10.1056/nejmoa2001017.

2. Rodon J, Noguera-Julian M, Erkizia I, et al. Search for SARS-CoV-2 inhibitors in currently approved drugs to tackle COVID-19 pandemia. , doi: 10.1101/2020.04.23.055756. 
3. Rejdak K, Grieb P. Adamantanes might be protective from COVID-19 in patients with neurological diseases: multiple sclerosis, parkinsonism and cognitive impairment. Multiple Sclerosis and Related Disorders. 2020; 42: 102163, doi: 10.1016/j.msard.2020. 102163.

4. Abreu G, Aguilar M, Covarrubias D, et al. Amantadine as a drug to mitigate the effects of COVID-19. Medical Hypotheses. 2020; 140: 109755, doi: 10.1016/j.mehy.2020.109755.
5. Tipton P, Wszolek Z. What can Parkinson's disease teach us about COVID-19? Neurol Neurochir Pol. 2020; 54(2): 204-206, doi: 10.5603/ pjnns.a2020.0039.

6. Cimolai N. Potentially repurposing adamantanes for COVID-19. Journal of Medical Virology. 2020; 92(6): 531-532, doi: 10.1002/jmv. 25752.

7. Smieszek S, Przychodzen B, Polymeropoulos M. “Amantadine disrupts lysosomal gene expression; potential therapy for COVID19". , doi: 10.1101/2020.04.05.026187. 